\title{
Verhindern Mundschutz und Handdesinfektion eine Influenzaepidemie?
}

\begin{abstract}
Am Beginn einer Influenzapandemie steht der passende Impfstoff häufig nicht in ausreichender Menge zur Verfügung. Zudem werden zunehmend Resistenzen gegen Virostatika beobachtet. Amerikanische Wissenschaftler prüften daher, ob sich die Ausbreitung der Influenza auch mit nicht pharmakologischen Mitteln eindämmen lässt.
\end{abstract}

- An der University Michigan in Ann Arbor (USA) wurden zu Beginn der Grippesaison 2007/2008 1176 Studenten aus 37 Wohnheimen an fünf Universitäten in drei Gruppen eingeteilt. Eine Gruppe sollte Mundschutz tragen und sich regelmäßig die Hände mit einer alkoholhaltigen Waschlotion desinfizieren. Die zweite Gruppe benutzte nur den Mundschutz und die dritte keines von beiden. Über sechs Wochen wurde registriert, wie oft grippeähnliche Symptome auftraten. Im Labor wurde dann geprüft, ob es sich tatsächlich um eine Influenza handelte

Eine maximale Reduktion von grippeähnlichen Symptomen konnte in der sechsten Versuchswoche in der Gruppe mit Mundschutz und Handdesinfektion registriert werden. Die Symptome waren hier um $75 \%$ seltener als in der Kontrollgruppe. Allerdings waren die Resultate wegen der insgesamt zu geringen Zahl an Infizierten statistisch nicht signifikant.

\section{Kommentar}

Dass der Schutzeffekt von Mundschutz und Handhygiene erst in der letzten Versuchswo- che voll zum Tragen kam, interpretieren die Studienautoren dahingehend, dass die Probanden zu Beginn des Versuchs die Handdesinfektion doch öfter vergaßen, diese aber im Laufe der Zeit zur Gewohnheit wurde. Die Kombination von Gesichtsmasken und Händehygiene hat - auch wenn hier keine statistische Signifikanz erzielt wurde - durchaus das Potenzial, die Verbreitung der Influenza einzudämmen. Diese nicht pharmazeutischen Maßnahmen sollten zu Beginn einer Influenzapandemie empfohlen werden.

K. MALBERG =

- A. E. Aiello et al.

(Department of Epidemiology, University of Michigan-School of Public Health, Ann Arbor, Michigan USA;E-mail: aielloa@umich.edu) Facemasks, hand hygiene, and influenza among young adults: A randomized intervention trial Published online on 25 January 2012 in PLoS ONE 7(1): e29744. doi:10.1371/journal. pone.0029744

\section{Achtung:}

\author{
Hier muss der \\ Dummy durch \\ eine Anzeige \\ ersetzt werden !!
}

